\title{
Hematoma pericárdico dos años después de cirugía coronaria
}

\author{
Rafael García Fuster, Rafael Llorens, J osé María Melero, J oaquín Barba*, \\ Salvatore di Stefano, J uan J osé Legarra y E duardo Alegría*
}

Servicios de Cirugía Cardiovascular y Torácica y *Cardiología. Clínica Universitaria.

Facultad de Medicina. Universidad de Navarra.

hematoma/ taponamiento cardíaco/ periodo postoperatorio/ cirugía cardíaca

E I taponamiento cardíaco tras cirugía cardíaca es una complicación que conlleva un riesgo elevado, que puede ocurrir tanto en el postoperatorio precoz como en el tardío. E I taponamiento tardío se ha definido, de forma arbitraria, como aquel que ocurre más allá del séptimo día del postoperatorio. Son mucho menos frecuentes que los precoces y han sido referidos, en la mayoría de los casos, hasta seis meses después de la intervención. Suelen plantear problemas diagnósticos que pueden repercutir negativamente en el pronóstico.

Por su atípica aparición, se presenta el caso de un paciente de 65 años que desarrolló un síndrome pospericardiotomía y posteriormente un hematoma pericárdico, tras casi dos años de una intervención de bypass aortocoronario. Se expone el caso y se realiza una revisión bibliográfica del taponamiento cardíaco postoperatorio tardío bajo un enfoque diagnóstico y terapéutico.

\section{PERICARDIAL HAEMATOMA TWO YEARS AFTER CORONARY SURGERY}

Cardiac tamponade is a life-threatening complication after cardiac surgery which may develop in the early or late postoperative period. The latest have been defined arbitrarily as the ones ocurring after the 7th postoperative day. They are less common than the early ones and most of the cases have been reported up to six months after the operation. They usually determine diagnostic difficulties that can negatively influence the prognosis.

Because of its atypical late appearance, a case of a 65 year old man is presented who developed a postpericardiotomy syndrome and subsequently a pericardial clot nearly two years after aortocoronary bypass grafting.

(Rev Esp Cardiol 1997; 50: 58-61)

\section{INTRODUCCIÓN}

El taponamiento cardíaco (TC) tras cirugía cardíaca es una complicación poco frecuente, pero con un riesgo elevado. Según las distintas series su incidencia oscila entre el $0,5 \%$ y el $5,8 \%{ }^{1,2}$. Su mortalidad se cifra en un $18 \%{ }^{2}$. La mayoría se presentan en la fase precoz desarrollándose en las primeras horas o días del postoperatorio; pero también pueden aparecer en los días subsiguientes. De forma arbitraria se ha definido el TC tardío como aquel que se presenta más allá del séptimo día del postoperatorio, habiéndose referido hasta los 6 meses tras la intervención ${ }^{2}$. Ajustándose a

Correspondencia: Dr. R. Llorens.

Servicio de Cirugía Cardíaca. Hospiten

Rambla Gral. Franco, 115. 38001 Santa Cruz de Tenerife.

Recibido el 5 de febrero de 1996

Aceptado para su publicación el 22 de marzo de 1996. esta definición su incidencia sería de un 0,3-2,6\%. El hecho de plantear dificultades diagnósticas puede repercutir negativamente en su pronóstico ${ }^{2}$.

Reseñamos un caso de aparición inusualmente tardía, tratando de aportar el enfoque diagnóstico y terapéutico que pensamos más apropiado.

\section{CASO CLÍNICO}

Varón de 65 años intervenido de triple bypass aortocoronario en enero de 1993, realizándose con vena safena a marginal izquierda, arteria mamaria interna izquierda a descendente anterior y mamaria derecha a la descendente posterior. El pericardio no fue cerrado al finalizar y se colocaron un drenaje retrosternal y otro pleural izquierdo, que fueron retirados a las 45 horas. El sangrado postoperatorio total fue de $1.045 \mathrm{ml}$. Fue dado de alta el octavo día con tratamiento antiagregante (aspirina $125 \mathrm{mg}$ diarios) que se mantuvo durante 22 meses. A los 5 meses de la intervención pre- 


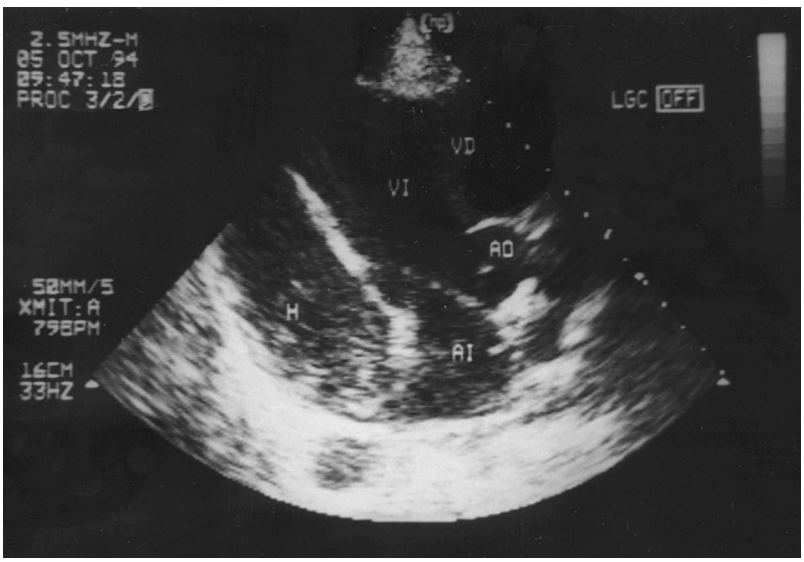

Fig. 1. Ecocardiograma transtorácico previo a la intervención (plano parasternal longitudinal); AD: aurícula derecha; AI: aurícula izquierda; H: hematoma; VD: ventrículo derecho; VI: ventrículo izquierdo.

sentó un síndrome pospericardiotomía con astenia y disnea progresivas. El electrocardiograma (ECG) mostraba bajo voltaje en cara frontal. En la radiografía de tórax (RxT) apareció un ligero derrame pleural bilateral sin cardiomegalia. En el ecocardiograma (ECO) se apreció un derrame pericárdico moderado en región inferoposterior y lateral con colapso de la aurícula derecha. La fracción de eyección (FE) calculada fue del $60 \%$. Tras tratamiento diurético y antiinflamatorio se evidenció una mejoría clínica con clara disminución del derrame y desaparición del bajo voltaje en el ECG.

En revisiones posteriores se confirmó la buena evolución, reseñando únicamente astenia crónica y un pequeño derrame pericárdico posterolateral encapsulado. Se diagnosticó de pericarditis postoperatoria con ligero derrame sin trascendencia clínica. A los 22 meses de la cirugía comenzó con disnea de esfuerzo progresiva llegando a ser de pequeños esfuerzos y roce pericárdico a la auscultación. De nuevo se objetivó la presencia de bajo voltaje en el ECG y ausencia de cambios en la RxT. En el ECO (fig. 1) apareció un derrame pericárdico sólido de localización posterior, con compresión de ventrículo izquierdo y función sistólica global ligeramente deprimida (FE 50\%). En el cateterismo realizado se objetivó en el ápex una zona de aspecto aneurismático, resultado de la compresión de la pared posteroinferior del ventrículo izquierdo y permeabilidad de todos los injertos. La presión capilar pulmonar era de $25 \mathrm{mmHg}$, la media en arteria pulmonar de $38 \mathrm{mmHg}$ y las presiones proto y telediastólicas de ambos ventrículos eran de 5 y $24 \mathrm{mmHg}$ para el izquierdo y 6 y $17 \mathrm{mmHg}$ para el derecho.

Se intervino en noviembre de 1994 (a los 23 meses de la cirugía de revascularización) con el diagnóstico de hematoma intrapericárdico retrocardíaco, con compresión de cavidades izquierdas e insuficiencia cardíaca grado III. Por toracotomía posterolateral izquierda se realizó evacuación del hematoma, encon-

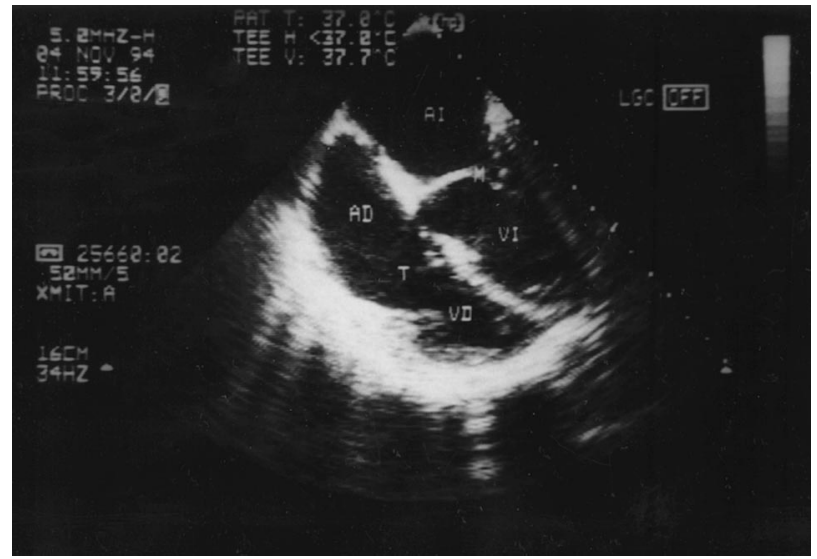

Fig. 2. Ecocardiograma transesofágico intraoperatorio postevacuación (cuatro cámaras); $A D$ : aurícula izquierda; $A D$ : aurícula derecha; M: mitral; T: tricúspide; VD: ventrículo derecho; VI: ventrículo izquierdo.

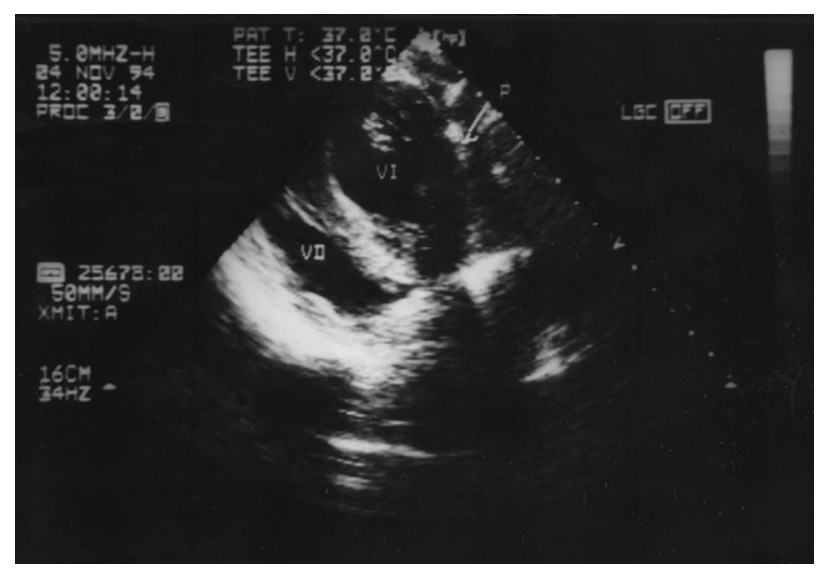

Fig. 3. Ecocardiograma transesofágico intraoperatorio postevacuación (plano transgástrico); P: pericardio; VD: ventrículo derecho; VI: ventrículo izquierdo.

trándose adherencias pleurales y pulmonares firmes sobre la cara lateral izquierda del pericardio y hematoma organizado entre dos de los injertos, con compresión severa de la cara posterolateral del ventrículo izquierdo. Una vez evacuado no se evidenció sangrado activo. Se practicó ECO (figs. 2 y 3) transesofágico intraoperatorio que demostró la recuperación de la contractilidad de la cara lateral del ventrículo izquierdo. El paciente presentó rápida mejoría clínica tras la cirugía. En los ECO posteriores se comprobó la completa evacuación del hematoma y clara mejoría de la función sistólica (FE 76\%).

\section{DISCUSIÓN}

El TC postoperatorio es una complicación importante pero poco frecuente $(0,5-5,8 \%)$ si lo comparamos con los derrames pericárdicos identificados mediante ECO bidimensional tras la cirugía cardíaca $(31-85 \%)^{2}$. Estos últimos no suelen tener repercusión 
clínica a diferencia de los primeros que acarrean una mortalidad de alrededor del $18 \%{ }^{2}$. Lo más frecuente es que aparezca en las primeras horas o días del postoperatorio inmediato (TC postoperatorio precoz), siendo fácil llegar al diagnóstico. El TC postoperatorio tardío fue descrito en primer lugar por Prewitt ${ }^{3}$. Se ha definido como el que ocurre a partir del séptimo día tras la operación. Se ha publicado poco acerca del margen temporal de aparición, siendo la mayoría de los casos referidos en la bibliografía de presentación previa a los 6 meses $^{2}$. Son escasos los que ocurren más allá de este tiempo, pudiendo destacar a $\mathrm{Sahni}^{4}$ que refiere 74 pacientes con TC postoperatorios tardíos ocurridos entre los 7 y 287 días de la intervención (media de 16) y Albat ${ }^{5}$ que presenta 2 casos, a los 3 y 10 meses tras cirugía valvular aórtica. Son extremadamente raros a partir del año, por lo que pensamos interesante plantear nuestro caso. Su interés estriba en que, al no establecerse una clara relación temporal entre el acto quirúrgico-TC, se pueden plantear problemas diagnósticos, máxime cuando estos taponamientos suelen tener una presentación atípica. Russo ${ }^{2}$ concluye que deberían considerarse en todo paciente que no progrese como cabría esperar, tras el alta.

$\mathrm{El}$ conocimiento de algunos factores de riesgo implicados nos debe hacer pensar en la posibilidad de un TC tardío $^{2}$. Se ha visto una clara relación con el uso de anticoagulantes y en recambios valvulares, aunque este no sea nuestro caso. Sí lo es, en cambio, la clara relación con el síndrome pospericardiotomía, responsable de derrames pericárdicos tardíos como resultado del proceso inflamatorio crónico: aquí la anticoagulación puede aumentar el riesgo de hemorragia intrapericárdica así como los salicilatos. Es habitual que produzcan compresión selectiva de cavidades debido a las adherencias pericárdicas, siendo común en la cara posterior, incluso cuando el pericardio no se cierra tras la cirugía. Esta localización también dificulta el diagnóstico. La compresión ejercida en nuestro paciente era selectiva sobre el ventrículo izquierdo, lo que puede justificar una aparición más tardía que si se afectara la aurícula, de fina pared y baja presión y, por tanto, pudiendo ocasionar un estado de bajo gasto rápidamente progresivo y precoz ${ }^{6}$. Los signos clásicos de TC suelen darse en derrames circunferenciales pudiendo estar ausentes en los regionales. El diagnóstico definitivo lo da la ECO y hay que apuntar la importancia de la ECO transesofágica en los de localización posterior ${ }^{7}$. El cateterismo cardíaco y coronariografía no aporta mayor información sobre la localización, pero clarifica la repercusión funcional. Además, en nuestro caso fue interesante con vistas a la cirugía evacuadora para verificar la permeabilidad de los injertos de la cirugía coronaria previa. El diagnóstico diferencial debe hacerse con la pericarditis constrictiva posquirúrgica, que es un fenómeno poco frecuente con una incidencia del
$0,2 \%-0,3 \%{ }^{8}$. Sus principales síntomas son disnea $(81 \%)$, dolor torácico $(34 \%)$ y astenia $(29 \%)$ y como signos físicos: edemas periféricos $(90 \%)$, aumento de la presión venosa yugular (86\%). Son útiles en el diagnóstico de esta pericarditis la tomografía computarizada y la resonancia magnética cardíaca, pero es requisito imprescindible para el diagnóstico la igualdad de las presiones diastólicas.

En lo que respecta al tratamiento, la localización, el tamaño y la consistencia (sólida o líquida) son factores determinantes. En los hematomas retrocardíacos abogamos por la toracotomía posterolateral izquierda, porque permite un buen abordaje, evitando las zonas de mayor adherencia y en caso de cirugía coronaria previa, disminuye el riesgo de lesión de los injertos. En nuestro caso, dada la localización y la naturaleza sólida de la colección, no se pudo efectuar pericardiocentesis ni drenaje por vía subxifoidea. Según $\mathrm{Sahni}^{4}$, la pericardiocentesis puede fracasar en derrames de localización posterior, en hematomas o en espacios pericárdicos menores de $20 \mathrm{~mm}$. Tampoco era aconsejable la esternotomía media, haciendo hincapié en la utilidad de la toracotomía izquierda ${ }^{5}$.

\section{CONCLUSIONES}

Podemos reseñar las siguientes conclusiones de nuestra valoración del tema:

a) A pesar de ser poco frecuente, hay que considerar la posibilidad de TC postoperatorio tardío en todo paciente intervenido de corazón que no evoluciona favorablemente. Debemos estar alerta ante clínica inespecífica (astenia, debilidad, anorexia, etc.). El síndrome pospericardiotomía puede ayudar a sospecharlo, sobre todo si se mantiene tratamiento anticoagulante. Pensamos que en estos pacientes sería aconsejable mantener una mayor vigilancia al menos durante los 18 meses siguientes.

b) Es habitual que aparezca en los primeros 6 meses postoperatorios, pero puede ocurrir también más tardíamente, incluso transcurrido el primer año.

c) La ecocardiografía es la técnica de elección en el diagnóstico, siendo sencilla y poco molesta para el paciente. La ecocardiografía transesofágica tiene una mayor sensibilidad en los hematomas de localización posterior.

d) La pontografía es aconsejable cuando se plantea evacuación quirúrgica en pacientes previamente intervenidos de coronarias. Verificar la permeabilidad de los injertos es crucial de cara a la intervención.

e) El mejor abordaje para los de localización posterior, máxime si la cirugía previa fue de coronarias, es la toracotomía izquierda.

f) La colocación de un drenaje retrocardíaco en el acto operatorio puede prevenir la aparición de colecciones sanguíneas de localización posterior. 


\section{BIBLIOGRAFÍA}

1. Wong PS, Pugsley WB. Raised international normalised ratio (INR): is it a cause or an effect of late cardiac tamponade? $\mathrm{Br}$ Heart J 1992; 68: 212-213.

2. Russo AM, O'Connor WH, Waxman HL. Atypical presentations and ecocardiographic findings in patients with cardiac tamponade ocurring early and late after cardiac surgery. Chest 1993; 104: 71-78.

3. Prewitt TA, Rackley CE, Wilcox BR, Scatliff JH, Young DT. Cardiac tamponade as a late complication of open heart surgery. Am Heart J 1968; 76: 139-141.

4. Sahni J, Ivert T, Herzfeld Y, Brodin LA. Late cardiac tamponade after open heart surgery. Scand J Thorac Cardiovasc Surg 1991; 25: 63-68.
5. Albat B, Picard E, Messner Pellenc P, Thevenet A. Late cardiac tamponade by localized compression of the left cavities after heart valve surgery. Arch Mal Coeur Vaiss 1991; 84: 1.3611.364 .

6. Nottestad SY, Mascette M. Loculated pericardial effusion and cardiac tamponade late after cardiac surgery. Chest 1992; 101: 852853.

7. Schoebrechts B, Herregods MC, Van de Werf F, De Geest H. Usefulness of transesophageal ecocardiography in patients with hemodinamic deterioration late after cardiac surgery. Chest 1993; 104: 1.631-1.632.

8. Cimino JJ, Kogan AD. Constrictive pericarditis after cardiac surgery: report of three cases and review of the literature. Am Heart J 1989; 118: 1.292-1.301. 